LBNL-43788

July 99

\title{
Holographic Cosmology and its Relevant Degrees of Freedom
}

\author{
RICHARD DAWID[ \\ Theoretical Physics Group \\ Ernest Orlando Lawrence Berkeley National Laboratory \\ University of California, Berkeley, California 94720, USA
}

\begin{abstract}
We reconsider the options for cosmological holography. We suggest that a global and timesymmetric version of the Fischler-Susskind bound is the most natural generalization of the holographic bound encountered in AdS and De Sitter space. A consistent discussion of cosmological holography seems to imply an understanding of the notion of "number of degrees of freedom" that deviates from its simple definition as the entropy of the current state. The introduction of a more adequate notion of degree of freedom makes the suggested variation of the Fischler-Susskind bound look like a stringent and viable bound in all 4-dimensional cosmologies without a cosmological constant.
\end{abstract}

\footnotetext{
aEmail: dawid@thsrv.lbl.gov
} 


\section{Introduction}

For quite some time the remarkable fact that the entropy of a black hole $(\mathrm{BH})$ is represented by its event horizon [1] has nurtured the suspicion that gravity in general could be holographic [2], meaning that all degrees of freedom (DOF) of a gravitational theory can be projected onto some lower dimensional surface(s) whose storage capacity is one bit information per

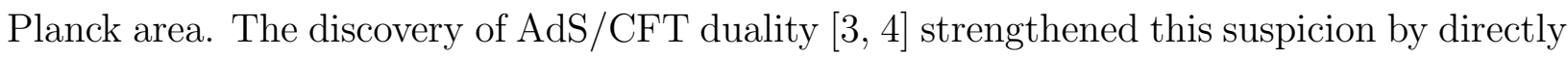
identifying a gravity theory on AdS with a lower dimensional field theory on its boundary. In [5] a specific realization of a general cosmological holographic principle was suggested. A very attractive point of that proposal is the fact that the suggested holographic bound is exactly saturated at the Planck time if one straightly extrapolates back from today's condition of the universe. Less satisfying seemed the fact that today the actual entropy inside the particle horizon is far lower than the FS-bound. The most serious problem however was the fact that in a closed universe the FS-bound seemed necessarily violated. During the last year various different modifications of the FS-conjecture have been formulated to deal with the described problems [6].

In this paper we reconsider the options for a cosmological holographic principle. In the first part we investigate what implications for a cosmological holographic principle one can infer from the realization of holography in AdS (section 2) and De Sitter space (section 3). We claim that the most natural generalization would be a principle that it is global, in the sense that it does not restrict the local density of DOF, and time symmetric.

Section 4 makes the statement, following the basic idea of [10], that a consistent discussion of cosmological holography requires a reconsideration of the notion of degree of freedom (DOF) in that context. The recent discussions of a cosmological holographic principle have been based on the assumption that the actual entropy of a system represents the number of degrees of freedom that have to be stored on some boundary in a holographic scenario. This is true as long as one deals with field theories in an equilibrium state disregarding gravity - in which case there is no holography anyway. However we argue that the assumption becomes quite questionable in a general cosmology. There are two aspects that change the situation. First a realistic cosmology is not in the highest entropy state because matter is more or less evenly distributed and gravitational clumping can enhance the entropy. It is not a priori clear whether the possible DOF must be identified with the actual entropy or the highest possible entropy. Second, the background as well as - if defined in some dynamical way - the position of the holographic boundary depend on the dynamics happening on that background. Therefore the validity of the background and the position of the bound- 
ary both can give restrictive conditions for the number of DOF. The central statement of this paper is that a definition of "degree of freedom" that takes into account the two aspects mentioned above seems to render a global and time-symmetric version of the original Fischler-Susskind conjecture viable and rather stringent for all four dimensional cosmologies with zero cosmological constant.

Section 5 describes the problems one encounters in general cosmological scenarios with a cosmological constant. We close with conclusions.

\section{Cosmological Holography}

The basic claim of a cosmological holographic principle is that some kind of holographic bound generally exists in cosmology. The upper bound for the number of degrees of freedom of a three-dimensional space-like or light-like space should be represented by the area of some two dimensional surface on which one bit information per Planck area can be stored.

The idea was first formulated in the early papers of t' Hooft and Susskind [2] and was more specifically realized by Fischler and Susskind in [5] (FS-conjecture). The conjecture goes as follows: Given some spherical spatial volume $V$ bounded by the surface $B$, all DOF entering through the future-lightcone that ends at $B$ can be stored on $B$ if $B$ can store one bit information per Planck area.

There exists one rather powerful argument in favour of the existence of a cosmological holographic principle along those lines: The corresponding holographic bound turns out to be valid in the past of our universe up to the Planck time and moreover seems to be more or less exactly fulfilled at the Planck time itself. This is a quite remarkable coincidence.

However on the other side the FS-bound has some problems.

First it seems that the bound is violated for a boundary $B$ around the center of a $\mathrm{BH}$ whose radius is smaller than the $\mathrm{BH}$-event horizon. Second it seems not very satisfying that today the actual entropy inside the particle horizon is far lower than the FS-bound. The most serious problem however is the fact that in a realistic closed universe the FS-bound seems necessarily violated. This has to do with the fact that in a matter dominated closed 4dimensional Robertson Walker spacetime light starting from one point at $t=0$ reaches the opposite pole on the sphere at the time of maximal expansion. Therefore already at times close to the maximal expansion there exist arbitrarily small $B$ s around the opposite pole which have to store nearly all of the universe's DOF, which seems impossible. 
During the last year various different modifications of the FS-conjecture have been formulated to deal with the described problems [6, 9, 7]. The most recent interesting attempt to construct a holographic principle that is generally valid was made in [7]. However the concept has some essential problems in connection with black holes which are partly discussed in [8]. In particular [8] found an explicit example where the bound of [7] does not hold.

As the situation stands today, it seems to us that no cosmological holographic principle is enforced by known gravity:

However a holographic principle might exist without being implied by semi-classical gravity. The one known example of a full holographic theory, AdS/CFT duality, was conjectured in the context of D-branes and does not follow from classical gravity. The suspicious frequency of holographic phenomena in connection with gravity really looks like a hint towards a general holographic principle that can only be understood in full quantum gravity. In that case this principle could well put new constraints on the cosmological evolution that do not exist in classical gravity. Without knowledge of quantum gravity the only way to understand in how far such a possibility is realistic is to check whether our universe as we know it is consistent with some holographic principle and possibly shows apparently unnatural constraints that can be understood in accordance with holography. This as we understand it is the spirit of the original work of Fischler and Susskind. In this paper we try to go a little further in that direction.

A good point of departure seems to be the known example of AdS/CFT duality. We look for a definition of cosmological holography that remains as close as possible to this example. If we compare the FS-conjecture or Bousso's covariant entropy conjecture with the situation in the case of AdS/CFT duality, we realize that the former are much stronger in a certain point. Since they allow for all possible $B^{\prime} s$, also very small ones, they give a bound on the local density of DOF, something AdS/CFT duality with the boundary strictly at infinity does not do. And it is exactly this requirement that produces considerable problems in connection with black holes. Additionally this requirement devaluates the strongest cosmological argument in favour of the FS-bound, namely the fact that the FS-bound is exactly saturated at the Planck time: If it was the case that the FS-bound for some reason holds even for the smallest boundary $B$ and the highest energy density anywhere in space, the statement that already the "naive" estimate in [5] leads to a saturated bound at the Planck time could only be judged as a mere accident.

\footnotetext{
${ }^{1}$ What seems to be true in all gravitational scenarios as long as one accepts the viability of statistics is the generalized second law formulated in [9]. This however is no holographic principle.)
} 
The arguments above lead us to the assumption that a cosmological holographic principle, if it exists, is likely to be a global principle in the sense that it does not give bounds on the local entropy density. Local realizations of holography can occur as a consequence of the global principle but do not necessarily hold under all circumstances.

To make the FS-bound global one just has to require that the surface $B$ must be the particle horizon respectively past event horizon of some observer. This definition makes the holographic conjecture immune against local problems with today's black holes. $B$ is small only close to the Planck time. The holographic conjecture therefore enforces the specific energy density of the universe one finds at the Planck time. It also enforces that the universe is smooth close to the Planck time avoiding high entropy states that include primordial black holes. Holography in this form therefore implies cosmic censorship.

\section{Pure De Sitter Space}

A main inspiration for the formulation of the holographic bound was AdS/CFT duality. However there is a fundamental difference between the static AdS scenario and a realistic cosmology: The AdS structure is only valid as long as the global energy density is zero. Otherwise the Einstein equations would enforce a different spacetime structure. Thus it is conceptually inconsistent to try to project states with finite global energy density onto the AdS boundary. Seen from that perspective the step to cosmological holography is the step to finite matter density.

Besides AdS (and Minkowski space that can be understood as it's infinite radius limit) there exists one more example for a zero matter density cosmology: De Sitter space. In this section we will discuss whether the principle of holography encountered in AdS and generalized to Minkowski space can also be implemented in De Sitter space. From this discussion we will get further hints concerning a consistent possible generalization of holography in a realistic cosmological context.

The spacelike dimensions of De Sitter space are closed which has some implications for the character of a holographic bound in this scenario. Since the space volume is finite,

\footnotetext{
${ }^{2}$ In inflationary scenarios the observed initial state is the state after re-heating. In this case one would hope to find an argument to replace the exponentially blown up and therefore quite meaningless particle horizon with an "effective" particle horizon reaching back to the endpoint of the exponential expansion.

${ }^{3}$ It seems to us that the connection between AdS/CFT and this condition has its subtleties. A discussion of that point is in preparation.
} 
the condition of background validity requires empty space. Any small matter contribution would change the background. While in the AdS case the holographic boundary of AdS sat at infinity, there is no infinity in de Sitter space. Therefore a holographic boundary will have to sit somewhere in space, thereby resembling already the situation we will encounter in finite matter density scenarios.

Let's have a closer look at the structure of De Sitter space. De Sitter space is the solution of the state equations

$$
\begin{aligned}
\frac{\Lambda}{3} R^{2}-1 & =\dot{R}^{2} \\
\Lambda R & =3 \ddot{R}
\end{aligned}
$$

Its metric can be written as

$$
d s^{2}=-d t^{2}+\alpha^{2} \cosh ^{2}\left(\alpha^{-1} t\right) d \Omega, \quad \alpha:=\left(\frac{3}{\Lambda}\right)^{1 / 2}
$$

Eq.(四) leads to the Hubble constant

$$
H:=\frac{\dot{R}}{R}=\left(\frac{\Lambda}{3}-R^{-2}\right)^{1 / 2}
$$

De Sitter space has a future and a past event horizon $E_{f}$ respectively $E_{p}$. They are defined as

$$
\begin{aligned}
& E_{f}(t):=R(t) \int_{t}^{\infty} \frac{d t^{\prime}}{R\left(t^{\prime}\right)} \\
& E_{p}(t):=\int_{-\infty}^{t} \frac{d t^{\prime}}{R\left(t^{\prime}\right)}
\end{aligned}
$$

Solving Eq.(1) gives

$$
R(t)=\frac{1}{H} e^{\left(H t-\frac{\ln 4}{2}\right)}+\frac{1}{4 H} e^{-\left(H t-\frac{\ln 4}{2}\right)}
$$

which leads to

$$
E_{f(p)}(t) / R(t)=\left.\frac{2}{H} \arctan \left[2 e^{\left(H t-\frac{\ln 4}{2}\right)}\right]\right|_{t(-\infty)} ^{\infty(t)}
$$


This implies

$$
\begin{aligned}
E_{f}(\infty)=E_{p}(-\infty) & =R(0)=H^{-1} \\
E_{f}(0)=E_{p}(0) & =\frac{\pi}{2 H}
\end{aligned}
$$

Therefore we have the following situation: At $t=-\infty E_{p}$ is at a distance $H^{-1}$ from the observer while $E_{f}$ is at the same distance from the opposite pole. They meet each other at $t=0$ at the equator and end up with exchanged roles at $t=\infty$. Each horizon travels a coordinate distance $\pi$ during its infinite lifetime. This situation is directly related to the AdS case. The corresponding statement there would be that light reaches infinity at $t=\pi / 2$.

It was shown in [13] that in the exponentially expanding phase of De Sitter space the area of $E_{f}$ plays the role of entropy exactly in the same way as in the case of the black hole. Since De Sitter space is empty, it would be natural to have a holographic bound that is exactly saturated by the geometric entropy. The holographic bound should resemble the area of the event horizon. Now the future event horizon is supposed to cover the DOF outside the horizon seen from the observers perspective. It therefore fits exactly as holographic bound around the point opposite to the observer's position on the sphere. The discussion above shows us that an area that resembles the future event horizon for one point represents the past event horizon $E_{p}$ for the point opposite on the sphere. Consequently $E_{p}$ is a natural candidate for the holographic bound on De Sitter space in the expanding phase.t.

Now, interestingly, in the contracting phase the whole situation is reversed. By changing exponential expansion to exponential contraction one changes the area $r=1 / H$ from representing $E_{f}$ to representing $E_{p}$. Since the definition of entropy does not depend on the time direction, now the De Sitter entropy must be defined by $E_{p}$. But the observer's $E_{p}$ is the opposite point's $E_{f}$. Therefore the holographic bound is represented by $E_{f}$. We get the remarkable result that the definition of the holographic boundary depends on the sign of the expansion of space. $E_{p}$ and $E_{f}$ change roles at the point of extremal extension. This point will play an important role in the discussion of cosmological holography.

In summary De Sitter space has a very natural holographic boundary that is consistent and

\footnotetext{
${ }^{4}$ Identical to the past event horizon in De Sitter space would be the outer apparent horizon that separates the anti-trapped region from the normal region around the other pole. This outer apparent horizon does not exist in open universes and therefore cannot serve as a general definition of a holographic boundary. It can however play a role in a definition of a holographic boundary together with the particle horizon. See [7].

${ }^{5}$ Like its cousins with negative respectively zero cosmological constant, the holographic boundary of De Sitter space has a constant area.
} 
stringent and resembles the global FS-bound in a time symmetric form. The closed structure of space in itself is not contradictory to holography.

\section{What is a Degree of Freedom}

We know that the scenarios with zero energy density are holographic. The way holography is realized there led us to the following concept for a possible general holographic principle: Assume $B$ to be an observer's past horizon in the expanding phase of the universe respectively an observer's future horizon in the contracting phase of the universe. Then all DOF which entered through the future light cone respectively will exit through the past light cone ending on $B$ can be stored on $B$ if $B$ can store one bit information per Planck area.

Next we have to check whether this holographic principle can hold in our real world. The crucial point will be how to deal with the closed universe scenario.

One question is of central importance in order to know what we are doing: We must have a clear understanding of what is a DOF. This turns out to be less trivial than it seems.

We saw already in De Sitter space that the background validity condition enforces empty space, which in turn is reflected by the fact that the holographic boundary is exactly saturated by geometric entropy. This is a first example showing that the validity of a holographic bound can be intricately entangled with cosmological restrictions.

In a realistic cosmology the situation is even more subtle. The evolution of space as well as the evolution of a holographic boundary in terms of comoving coordinates depends on the matter and radiation energy in that space. But to make the statement what DOF can be stored on a given surface, it is necessary first to know where this surface is. Holography can only talk about DOF under the precondition that the boundary is where it is. Therefore the full history of the universe leading up to the current position of $B$ has to be considered in deciding which DOF are to be stored on $B$.

In order to deal with this complication we introduce the notion of "relevant degree of freedom": Assume some cosmology and some holographic boundary surface put on the spacetime of that cosmology. Those states of the system which are consistent with the given evolution of both the universe and the holographic boundary are called relevant DOF for that boundary. Only relevant DOF are to be stored on a holographic boundary.

Note that the relevant DOF for a boundary are still to be distinguished from those which can be stored on that boundary. The question which subset of the relevant DOF eventually 
are accounted for by the holographic boundary depends on the specific definition of the holographic conjecture.

How exactly to define "consistent with the evolution" in the definition above is not quite clear. The closer one looks, the less tractable situation becomes. The dynamics of the holographic boundary depends not just on the global features of spacetime but also to some extent on the local conditions at a given point. The problem is reminiscent of those essential problems in gravity connected to the fact that what one would like to use as background space is actually part of the dynamics. The only way to deal with this situation at the moment seems to be to neglect small local effects and just look for intuitively plausible global restrictions.

We consider a flat spacetime evolving from a big bang according to Einstein's equations and look at the situation at some time $t$. What are the relevant DOF of this system? The global structure of spacetime is defined by the initial parameters of the cosmology. Higher energy density would imply a closed space, lower energy density an open space. Both would imply a different size and position of $B$ which means that both cannot represent relevant DOF of any $B$ in flat space.

The position of $B$ won't be influenced by the degrees of freedom which represent the entropy of the state (at least to a good approximation). Therefore those must be counted as relevant DOF. The crucial question is the following: Is the number of relevant DOF inside a surface $B$ represented by the actual entropy of the system or by the maximal entropy state possible. Before dealing with the question we have to make an assumption about the maximal entropy state of the system. Given a certain energy content, the highest possible entropy will be connected to the largest possible black hole. Since holography enforces an initial state without black holes, all black holes must form dynamically and thus cannot be arbitrarily large. We assume that the maximal entropy state corresponds to a black hole with the mass

$$
M_{\max }(t) \sim \rho(t) H^{-3} \sim \rho(t) E_{p}^{3}
$$

where $\rho(t)$ is the global energy density. The maximal entropy inside $B$ therefore should be represented by a $\mathrm{BH}$ that contains all energy inside $B$. The example of AdS suggests that the DOF of this system should be stored at the boundary. However the situation requires closer examination: Holography forces the initial state to be a smooth state that has no black holes and whose radiative entropy is in equilibrium. If one treats the system classically, all evolution is strictly deterministic, therefore the number of DOF in a system has to remain constant. At a later stage after black holes have formed this would mean that a big number 
of BH states would be locally allowed but do not stem from a purely radiative initial state. Thus they are not relevant DOF. This argument would reduce the number of relevant DOF to the value of radiative entropy.

Quantum processes however do produce new DOF. An extreme example is inflation where the quantum process of re-heating produces basically all DOF of today's world. Assume there exists an initial microstate at the Planck time that leads to a single black hole storing all matter inside $B$ at time $t$. One would expect, without making any more specific statement about BH-physics, that eventually all BH states can be reached through quantum tunneling in the BH. Therefore one should conclude that all BH DOF are relevant. This does not yet mean that they are all to be accounted for at the boundary. The FS-condition says that only those DOF are to be stored which enter through the horizon. If a $\mathrm{BH}$ is develops inside the horizon and new BH DOF are produced, these DOF cannot be stored at the horizon. However every BH at any moment can be thought of as entering some particle horizon around an observer somewhere else. We conclude that the holographic bound has to cover a number of DOF whose order of magnitude is somewhere close to the entropy of the maximal $\mathrm{BH}$.

How does this apply to the actual universe? A rough estimate says that the maximal BH entropy inside the particle horizon today is

$$
S_{\max }(\text { today }) \sim 10^{123}
$$

while the area of the horizon in Planck units is

$$
A_{H}(\text { today }) \sim t(\text { today })^{2} \sim 10^{122}
$$

The bound looks realistic and plausibly saturated. The next question is in how far this remains the case in the past respectively the future.

The radius of the particle horizon moves like

$$
r_{P H}(t) \sim t
$$

In the matter dominated regime the line element grows in $d$ spatial dimensions like

\footnotetext{
${ }^{6}$ Note that this is fundamentally different from the case of DOF production through re-heating. The latter is an isotropic process that looks the same for any particle horizon. Consequently the newly produced DOF inside the blown up particle horizon are truly "invisible" to the holographic boundary.
} 


$$
R(t) \sim t^{\frac{2}{d}}
$$

and we have a constant energy per comoving volume

$$
\rho(t) R^{d}(t)=K
$$

This means that the energy inside the horizon grows like

$$
M_{P H} \sim \rho(t) r_{P H}^{d}(t) \sim K t^{d-2}
$$

On the other hand the $\mathrm{BH}$ mass goes like

$$
M_{B H} \sim r_{B H}
$$

and the BH entropy goes like

$$
S_{B H} \sim r_{B H}^{d-1}
$$

If we identify the maximal entropy $S_{\max }$ with the black hole built from all energy inside the horizon, we see that

$$
\frac{S_{\max }(t)}{A(t)} \sim t^{(d-3)(d-1)}, \quad \text { for } \quad t>t_{D}
$$

where $t_{D}$ is the decoupling time from which on the dynamics is matter dominated. For $d=3$ one gets

$$
\frac{S_{\max }^{d=3}(t)}{A^{d=3}(t)}=\text { const }, \quad \text { for } \quad t>t_{D}
$$

This means that if saturated today, the holographic bound remains saturated for all time into the future and back until $t_{d}$.

One has to note that this nice result is only true for 3 spatial dimensions. In particular $\frac{S_{\max }(t)}{A(t)}$ increases with a positive power of $t$ for $d>3$ and thus necessarily leads to a violation of the holographic bound at some time.

The case of radiation domination for $d=3$ with 


$$
R(t) \sim t^{\frac{1}{2}}
$$

leads to

$$
\frac{S_{\max }(t)}{A(t)} \sim t, \quad \text { fort }<t_{d}
$$

Therefore $\frac{S_{\max }(t)}{A(t)}$ would decrease if one goes further into the past beyond $t_{d}$. However in that regime it clearly makes no sense to consider $\mathrm{BH}$ entropy as relevant DOF since radiation domination is conceptually exactly opposite to $\mathrm{BH}$ domination.

The case of an open universe initially cannot be distinguished from the flat case. At late stages the ratio $\frac{S_{\max }(t)}{A(t)}$ will become considerably smaller than in the flat case. Assuming that the holographic bound is saturated in the flat case, in the open case the bound will be nearly saturated at the early stages of matter domination while the number of DOF will fall below the saturation level later.

The most interesting scenario is the closed universe. This is where a holographic bound is most difficult to define. In [5] where the degrees of freedom were identified with the actual entropy of the universe at a given state, it was found that a holographic bound defined by the particle horizon necessarily is violated at late stages when the boundary has entered the opposite half of the spatial sphere, therefore decreasing its coordinate area until, when it reaches the opposite point, it eventually becomes zero. In the discussion of the flat case we argued that the current entropy underestimates the relevant DOF. Now we will argue that in the closed case it is a radical over-estimation.

At this point we have to remember the time symmetric form of our holographic conjecture. The definition of the holographic bound is connected to the sign of the universe expansion. The holographic bound for the contracting phase is given by the future event horizon. We have seen that holography enforces a smooth initial state without initial black or white holes. Now this implies that we also must have a smooth final state. But this means that existing black or white holes have to dissolve in the contracting phase. The entropy arrow must be reversed there. Holography connects the entropy arrow to the sign of the universe expansion.

What does this mean for the DOF? Let us consider a certain macrostate at the time of maximal expansion $\left(t_{\max }\right)$. The microstates of this macrostate represent the entropy at that stage. Now due to the time symmetric nature of holography, relevant DOF are only those which are consistent with the universe evolution seen from both time directions. In other words the requirement that the system has to return to a lower entropy state at the final 
state has to be fulfilled by each state that represents a relevant DOF. This however will rule out most of the microstates at time $t=t_{\max }$. Only the small subset that leads back to the initial condition represents relevant DOF. The consequences are clear. It is not justified to use the entropy as a measure for relevant DOF in a closed universe. Let us see how this fits into the cosmological picture.

The evolution of a closed $(d+1)$-dimensional spacetime goes like

$$
R(\chi) \sim(\sin |\kappa \chi|)^{\kappa^{-1}}, \quad \kappa=\frac{d}{2}(1+\gamma)-1
$$

where $\chi$ is the coordinate position of the particle horizon,

$$
\chi(t):=\int_{0}^{t} \frac{d t^{\prime}}{R\left(t^{\prime}\right)}
$$

and $\gamma$ is the ratio between pressure and matter density. In 3 spatial dimensions we therefore have the following situation: In the matter dominated case with zero pressure we have $\gamma=0$ and $\kappa=1 / 2$. At the time of maximal expansion, characterized by $\kappa \chi=\pi / 2$ we have $\chi=\pi$. The particle horizon reaches the opposite pole at the time of maximal expansion. In the contracting phase the future event horizon plays the role of the holographic bound making the situation time symmetric. Since holography enforces an initial radiation dominated state in fact the holographic boundary will not quite reach the pole, meaning that at the time of maximal extension there still remains a small area for the boundary. This fits nicely into the picture described above.

In a scenario where the universe remains radiation dominated throughout its evolution, we have $\gamma=1 / 3$ so that $\kappa=1$ and $\kappa \chi=\chi$. The horizon just reaches the equator at the point of maximal expansion. Therefore the bound at the time of maximal expansion is not much tighter than it would be at the same time in a flat scenario. If the initial state is purely radiative, the radiative entropy can be stored at the boundary throughout the whole evolution of the universe.

If we understand radiation domination in its extreme realization, we have have a situation where gravitational clumping does not happen at all. There is no gravitational entropy. The radiative (as the much lower baryonic) entropy however is in an equilibrium state from the

\footnotetext{
${ }^{7}$ It is highly nontrivial to formulate this statement more exactly in the framework of quantum statistics (See some considerations in [10]). In this paper we will not go deeper into that matter.
} 
start, therefore the initial and final conditions do not pose any restrictions. This means that the radiative entropy in this case in fact represents the relevant DOF, which again nicely fits into the picture.

One has to notice that this nice situation once again only holds for $d=4$. The defined holographic principle seems inconsistent with lower dimensional cosmologies while for higher dimensional cosmologies the holographic bound evolves less far on the sphere and therefore does not fully reflect the constraints due to recontraction.

\section{Cosmological Constant plus finite Energy Density}

In this chapter we have a short look at the most general case of cosmologies where a cosmological constant $\Lambda$ is joined by finite energy density. In that case the statement that the universe is closed and the statement that it recontracts do not imply each other. Therefore the nice connection between the shrinking of the the holographic boundary around the other

pole and the reduction of relevant DOF due to recontraction does not work any more.

A cosmology with negative $\Lambda$ always recontracts. Therefore one would expect a reduction of relevant DOF similar to the closed cosmology with $\Lambda=0$. However cosmologies with negative $\Lambda$ can be flat or open so that the boundary does not shrink. It seems that the holographic bound is unreasonably high.

The situation in case of positive $\Lambda$ is even worse. The problematic case is the closed scenario. Let us assume a matter content sufficient to make the universe recontract. If we ad a small $\Lambda$, the effect will be to drag out the large expansion phase before recontraction takes over. This means that the particle horizon will - in the matter dominated case - reach the opposite pole earlier than the time of maximal expansion and vanishes. This would imply that there are no DOF at all. This is most difficult to understand if $\Lambda$ is slightly above the value necessary to prevent recontraction. In that case there will be a phase of semi-stability at a certain expansion followed by the transition to exponential expansion. The particle horizon will hit the other pole in the semi-stable phase even though the restrictions on relevant DOF stemming from recontraction do not apply. For even higher $\Lambda$ we have the case of a bouncing universe looking similar to pure De Sitter space. In that case the horizons that should represent the holographic bound also vanish at some time at the point on the sphere opposite to the observer. 


\section{Conclusions}

We have formulated what we believe to be the most straightforward generalization of the holographic bound one encounters in cosmologies with zero matter density. We further argued that the DOF relevant for holography are not simply the ones represented by the actual entropy. The resulting picture of a global time-symmetric holographic principle and a set of relevant DOF does not fit into most cosmological scenarios with finite energy density. The holographic bound fails for a flat universe in more than 4 spacetime dimensions, for a closed universe in less than 4 spacetime dimensions and for a closed scenario in 4 dimensions with a considerable positive cosmological constant. On the other side the bound it imposes is far higher than necessary for most of the other scenarios.

However there is one scenario where the holographic bound is both viable and stringent. This scenario is the 4-dimensional universe with a strongly suppressed cosmological constant we actually live in. It is surely too early to judge whether this is just an accident or not. If it is more than an accident, it would mean that holography hints towards an excitingly strong restrictiveness of full quantum gravity.

Acknowledgments: We would like to thank Paolo Aschieri for very useful discussions. This work was supported in part by the Director, Office of Science, Office of High Energy and Nuclear Physics, Division of High Energy Physics,, of the U.S. Department of Energy under Contract DE-AC03-76SF00098 and in part by the Erwin Schrödinger Stipendium Nr. J1520-PHY. 


\section{References}

[1] J. D. Bekenstein, Phys. Rev. D7, (1973) 2333;

S. W. Hawking, Commun. Math. Phys. 43, (1974) 199

[2] G. t' Hooft, gr-qc/9310026;

L. Susskind, J. Math. Phys. 36 (1995) 6377, hep-th/9509089.

[3] J. Maldacena, hep-th/9711200;

S.S. Gubser, I.R. Klebanov and A.M. Polyakov Phys. Lett. B428 (1998) 105, hep-th/9802109;

E. Witten, hep-th/9802150.

[4] O. Aharony, S. S. Gubser, J. Maldacena, H. Ooguri, Y. Oz, hep-th/9905111

[5] W. Fischler and L. Susskind, hep-th/9806039

[6] D. Bak and S. J. Rey, hep-th/9811008; hep-th/9902173;

A. K. Biswas, J. Maharana and R. K. Pradhan, hep-th/9811051;

G.Veneziano, Phys. Lett. B454, (1999) 22, hep-th/9902126; hep-th/9907012;

N. Kaloper and A. Linde, hep-th/9904120;

R. Brustein, gr-qc/9904067;

R. Brustein, S. Foffa, R. Sturani, gr-qc/9907032

[7] R. Bousso, hep-th/9905177; hep-th/9906022

[8] D. A. Lowe, hep-th/9907062

[9] R. Easther and D. A. Lowe, Phys. Lett. 82, (1999) 4967, hep-th/9902088

[10] R. Dawid, Phys. Lett. $B 451$ (1999) 19, hep-th/9811243

[11] L. Susskind and E. Witten, hep-th/9805114

[12] E. Witten, Adv. Theor. Math. Phys. 2 (1998) 505, hep-th/9803131

[13] G. W. Gibbons and S.W. Hawking, Phys. Rev. D15 (1977) 2738 